Check for updates

Cite this: RSC Adv., 2017, 7, 56256

Received 13th September 2017 Accepted 29th November 2017 DOI: $10.1039 / \mathrm{c} 7 \mathrm{ra09738g}$

rsc.li/rsc-advances

\section{PTH coatings on titanium surfaces improved osteogenic integration by increasing expression levels of BMP-2/Runx2/Osterix}

\author{
Kaichen Lai, (D) ${ }^{a}$ Yue Xi, ${ }^{a}$ Xiaoyan Miao, ${ }^{b}$ Zhiwei Jiang, ${ }^{a}$ Ying Wang, ${ }^{c}$ Huiming Wang ${ }^{d}$ \\ and Guoli Yang (iD *a
}

The aim of this experiment was to assemble parathyroid hormone (PTH) coatings on titanium surfaces and evaluate the effect on implant osseointegration. PTH was assembled on surfaces of titanium with different layers $(5,7$, and 10$)$ using a layer-by-layer assembling technique. The degradation rate of PTH coatings was evaluated by measuring the concentration of PTH release. Surface analysis using $\mathrm{X}$-ray photoelectron spectroscopy (XPS), field emission scanning electron microscopy (FSEM), and surface contact angle measurements revealed a multilayer structure and confirmed that PTH layers could be assembled on titanium. Bone mesenchymal stem cells (BMSCs) cultured on the multiple layer coatings displayed significantly higher alkaline phosphatase activity (ALP) on the 7th day. The osteocalcin concentration was increased significantly in the 7-layer and 10-layer groups. Expression of bone morphogenetic protein 2 (BMP-2), runt-related transcription factor 2 (Runx2), and Osterix were up-regulated at day 7 and day 14. This showed that incubation with PTH enhanced cell proliferation to a statistically significant extent compared with the control group. Results of in vivo experiments demonstrated the formation of new bone, bone-implant contact (BIC), and the expressions of BMP-2, Runx2, and Osterix in the PTH-loaded group were enhanced. It was concluded that PTH coatings may have the potential benefit of enhancing implant osseointegration.

\section{Introduction}

Osteoporosis is a systemic disease from which more than 200 million people suffer worldwide. ${ }^{\mathbf{1} 2}$ It leads to decline of bone mineral density (BMD) and an increase of fracture risk. Good primary stability is essential for long-term implant success. ${ }^{3} \mathrm{~A}$ large retrospective cohort analysis identified osteoporosis as a significant variable associated with early dental implant failure. ${ }^{4}$ Therefore, there has been an increasing interest in improving implant osseointegration in osteoporotic patients.

During the past several decades, various methods have been developed to achieve quick osseointegration in osteoporosis patients, including electrochemical deposition, ${ }^{5}$ calcium phosphate coatings, ${ }^{6} \mathrm{TiO}_{2}$ nanoporous modification, ${ }^{7}$ and strontium hydrothermal treatment. ${ }^{8}$ Furthermore, a novel research field

\footnotetext{
${ }^{a}$ Department of Implantology, Stomatology Hospital, School of Medicine, Zhejiang University, Yan'an Road, Hangzhou, China.E-mail: 7308037@zju.edu.cn

${ }^{b}$ Department of Science and Education, Stomatology Hospital, School of Medicine, Zhejiang University, Yan'an Road, Hangzhou, China

${ }^{c}$ Department of Oral Medicine, Stomatology Hospital, School of Medicine, Zhejiang University, Yan'an Road, Hangzhou, China

${ }^{d}$ Department of Oral and Maxillofacial Surgery, Stomatology Hospital, School of Medicine, Zhejiang University, Hangzhou, China
}

investigating the addition of peptides to implant surfaces has emerged in implant dentistry.

It has been reported that implant surfaces with Arg-Gly-Asp (RGD)-peptide had a positive effect on promoting differentiation and mineralization of osteoblastic progenitor cells. ${ }^{9}$ It has been confirmed that the P-15 peptide coated surface led to higher BIC and greater peri-implant bone density. ${ }^{10}$ Yoo investigated implant surfaces with poly(lactide-co-gly-dolide) (PLGA) and BMP-2 facilitated osseointegration in an early stage. ${ }^{\mathbf{1 1}}$

Parathyroid hormone ( $\mathrm{PTH})$ is produced by the parathyroid glands and plays a key role in regulating phosphate and calcium metabolism. ${ }^{12}$ It has been reported that PTH might enhance bone formation by promoting osteoblast growth and decreasing osteoblast cell death or apoptosis. ${ }^{\mathbf{1 3 4}}$ Studies confirmed an anabolic effect with a synthetic PTH fragment in promoting fracture healing. ${ }^{\mathbf{1 5}, 16}$ In recent years, the effect of PTH in enhancing osseointegration has been investigated. ${ }^{17,18}$ However, injection treatment was used in most of studies. Few studies have focused on bone regeneration enhancement of local PTH delivery system.

Multiple methods were used to assemble peptides on titanium surfaces. Hydroxyapatite (HA) plasma spray coating is a well-established method. It has been a common carrier for peptide assembling. ${ }^{19}$ Peptides can be adsorbed to titanium surfaces by physical methods. ${ }^{20}$ Calcium phosphate coatings 
were used to adsorbed PTH on titanium surface. ${ }^{21}$ However, it can not get long-term release.

The layer-by-layer (L-B-L) assembling technique is used to assemble a large amount of materials on different surfaces. ${ }^{22}$ Compared LBL assembling technique to the former methods, it is much milder, simpler, more immediate, less cost, and it can release drugs pulse for a long time. ${ }^{23}$ It would not lead to strong protein denaturation and would not be restricted to a single monolayer. What is more, the exploitation of LBL assembling technique to create materials for sensing, storage, chemical transformation permeation, and delivery offers a wide range of applications for biotechnologies or biomedical use..$^{24,25}$ Moreover, our group is familiar with this method and has successfully assembled RGD-peptides on the roughed titanium implants surface. ${ }^{26}$

The aim of the present experiment was to assemble PTH coating on titanium surfaces with the LBL assembling technique and evaluate the effects on osteogenic integration.

\section{Materials and methods}

\subsection{Titanium plate preparation}

Plates $(8 \mathrm{~mm} \times 8 \mathrm{~mm} \times 1.5 \mathrm{~mm})$ were prepared as in the previous method reported by Yang et al. ${ }^{27}$ In short, the plates were polished, blasted and ultrasonic washed by acetone, $75 \%$ ethyl alcohol and double-distilled water for 15 minutes, respectively. After that, samples were managed with a solution which contains $0.11 \mathrm{M} \mathrm{HF}$ and $0.09 \mathrm{M} \mathrm{HNO}_{3}$ for 10 minutes at room temperature and then 30 minutes was needed to treat samples with a solution consisting of $5.80 \mathrm{M} \mathrm{HCl}$ and $8.96 \mathrm{M}$ $\mathrm{H}_{2} \mathrm{SO}_{4}$ at $80^{\circ} \mathrm{C}$. Samples were cleaned by distilled water again and blown dry with nitrogen.

\subsection{Preparation of the coating}

The L-B-L assembling technique is based on the attraction between opposite charges depositing in alternate layers. The process involves the following steps.

(1) Clean the plate and immerse it into solution of chitosan (5 mg mL ${ }^{-1}$ ) (Yunzhou Biochemistry, Qingdao, China) in $0.2 \%$ acetic acid for 20 minutes to produce a surface with positive charges and then rinse it with water.

(2) Dip the plate into solution of solution of hyaluronic acid (HA) $\left(0.5 \mathrm{mg} \mathrm{mL}^{-1}\right)$ (Sigma, USA) for 5 minutes and then rinse it with water. A layer containing negative charges on the surface of substrate is formed by adsorption.

(3) Dip the substrate into PTH solution. The positively charged peptides are adsorbed onto the negatively charged surface and bonded to the surface of titanium plate.

(4) Remove the substrate and rinse it with water and 1 layer is successfully assembled.

Repeat steps (2)-(4) in a loop and the coating containing PTH can be obtained.

\subsection{Surface characterization}

The surface morphological structure was examined by field emission scanning electron microscopy (FSEM, SU8010,
Hitachi, Japan). X-ray photoelectron spectroscopy (XPS) was used to examine the chemical ingredient of the coating. The wet ability of coating was examined with an angle contact metering system (SL200B, Solon Tech. Inc. Ltd. Shanghai, China).

\subsection{Release concentration analysis}

The PTH coated titanium plates were put into $1 \mathrm{~mL}$ of phosphate buffer solution (PBS) in a 24-well plate and the temperature was maintained at $37^{\circ} \mathrm{C}$. At each given time point, $0.5 \mathrm{~mL}$ of PBS was collected into a microcentrifuge tube and stored at $-20{ }^{\circ} \mathrm{C}$ as the test sample, and then $0.5 \mathrm{~mL}$ of fresh PBS was added to the plate. The given timings were 0.5 hour, $1 \mathrm{~h}, 2 \mathrm{~h}, 3 \mathrm{~h}$, $5 \mathrm{~h}, 24 \mathrm{~h}, 2$ days, $3 \mathrm{~d}, 5 \mathrm{~d}, 7 \mathrm{~d}, 10 \mathrm{~d}, 14 \mathrm{~d}, 17 \mathrm{~d}, 21 \mathrm{~d}, 24 \mathrm{~d}$ and $28 \mathrm{~d}$.

The protein sequence of PTH contains tryptophan whose fluorescence is excited when the wavelength is $280 \mathrm{~nm}$. Therefore, the release concentration can be examined by a microplate reader.

\subsection{Isolation and culture of rat BMSCs}

This study was approved by The Institutional Animal Care and Use Committee of Zhejiang University, Hangzhou, China according to "Experimental Animal Management Ordinance" issued by Chinese science and technology commission and "Laboratory Animal Science Guidance" issued by Chinese science and technology ministry. Male Sprague-Dawley rats (age: 3 weeks old) were used in this study. The procedure of isolation and culture of the BMSCs were executed as previously described..$^{28}$ BMSCs were cultured in $\alpha$-MEM (Jima, China) with $10 \%$ fetal bovine serum (Gibco, USA) and $100 \mathrm{U} \mathrm{mL}^{-1}$ streptomycin and penicillin supplemented in a $5 \% \mathrm{CO}_{2}$ at $37{ }^{\circ} \mathrm{C}$ as described. The third generations of BMSCs were used in the experiment.

\subsection{Cell proliferation}

Cell proliferation was detected by measuring the increase in BMSCs with a Cell Counting Kit-8 (Donjindo, Japan). At day 1, day 3 and day 7 after removing the medium, cells were covered by medium containing $10 \%$ CCK8 and were incubated at $37{ }^{\circ} \mathrm{C}$ in a $5 \% \mathrm{CO}_{2}$ circumstance with atmosphere humidified for 3 hours. The fluorescent intensity of the medium was measured with a microplate spectrophotometer (Spectra M2, Molecular, USA) at an excitation wavelength of $405 \mathrm{~nm}$. BMSC numbers were determined by measuring the optical density against a standard curve.

\subsection{Alkaline phosphatase (ALP) activity assay}

The ALP activity of BMSCs was detected at days 7 and 14 with a phosphatase substrate kit (Wako, Japan), which measures the release of $p$-nitrophenol from $p$-nitrophenylphosphate at $\mathrm{pH}$ 10.2 and normalized by cellular total protein. Cellular total protein was detected with a bicinchoninic acid (BCA) protein assay kit (Biyuntian, China). The expression of ALP activity was nanomole of $p$-nitrophenol released per microgram of total cellular protein per hour. 


\subsection{Osteocalcin release assay}

Osteocalcin was one of extracellular matrix proteins which released into medium and was measured at days 7 and 14 by Osteocalcin EIA Kit (R\&D System Inc, USA). A spectrophotometer was used to read the absorbance at $450 \mathrm{~nm}$. Results were expressed as ng mouse osteocalcin in the medium per milligram of total cellular protein.

\subsection{Real-time reverse transcription-polymerase chain reaction (RT-PCR) analysis}

According to the results of surface characterization analysis, cell proliferation and differentiation ability analysis, the most suitable group was chosen from the three experimental groups for RT-PCR analysis. After osteoblastic induction for 7 and 14 days, total RNA was extracted from BMSCs incubated on titanium plate of different groups. Reverse transcription was conducted following closely to generate cDNA with PrimeScript RT Reagent Kit (Takara, Dalian, China). Real-time PCR was detected using a SYBR Green Kit (Takara, Daian, China) with an ABI7500 Prism Sequence Detection System (Applied Biosystems, Foster City, CA). The sequences of PCR primers for BMP-2, Runx2, and Osterix (Sangon Biotech, Shanghai, China) were presented in Table 1 . The $2^{-\Delta \Delta C_{\mathrm{T}}}$ method was used to calculate the expression of target genes.

\subsection{Titanium implants and surface treatment}

The shape of titanium implants used in this study were screw ( $n$ $=$ 108). The implant length was $6.0 \mathrm{~mm}$ and the outside diameter was $2.2 \mathrm{~mm}$. The space was $1.2 \mathrm{~mm}$ between each thread with a depth of $0.2 \mathrm{~mm}$. The preparation of titanium implants was as same as the titanium plate. In the in vivo study, assembly of 7-layer PTH was chosen as the experimental group.

\subsection{Animal experiment}

Fifty-four female Sprague-Dawley rats, aged 3 months, were assigned to three experimental groups.

The establishment of osteoporotic animal model needed 12 weeks after bilateral ovariectomy. Subsequently, two different implants were placed into the medial aspect of the distal tibial close to metaphysis of every rat. Implant placement was performed under general anesthesia (10\% chloral hydrate solution, $0.3 \mathrm{~mL} / 100 \mathrm{~g}$, administered intraperitoneal and supplemented when it was necessary). Before the operation, the surgical area

Table 1 Nucleotide sequences for RT-qPCR primers

\begin{tabular}{ll}
\hline Gene & Sequences of primer $\left(5^{\prime}-3^{\prime}\right)$ \\
\hline GAPDH, rat & Forward: GGCACAGTCAAGGCTGGAATG \\
& Reverse: ATGGTGGTGAAGACGCCAGTA \\
BMP-2, rat & Forward: ACAAACGAGAAAAGCGTCAAGC \\
& Reverse: CCCACATCACTGAAGTCCACATA \\
Runx 2 , rat & Forward: CAGTATGAGAGTAGGTGTCCCGC \\
& Reverse: AAGAGGGGTAAGACTGGTCATAGG \\
Osterix, rat & Forward: CTGGGAAAAGGAGGCACAAAGA \\
& Reverse: GGGGAAAGGGTGGGTAGTCATT
\end{tabular}

was received lidocaine injection locally. An incision was made aseptically over the anteromedial tibias, and overlapping muscles were dissected bluntly to expose metaphysis. Holes were prepared and enlarged to a diameter of $2.2 \mathrm{~mm}$ sequentially by intermittent drilling with a low speed of rotary and persistent saline watering. The blank, HA and PTH implants were placed in the holes until the tip of the implant reached the bone cortex on the other side. Two implants from different groups were inserted in one animal randomly. After implantation, intramuscular injection of antibiotics (penicillin 400000 IU per day) was performed for 3 days. At 1 week and, 2 and 4 weeks after the operation, animals were sacrificed. Half of the tibias were gathered for micro-CT analysis and histomorphometric evaluation and the rest were saved for histological evaluation.

\subsection{Micro-CT analysis}

The tissues involving implants were retrieved and fixed in $4 \%$ paraformaldehyde solution for $24 \mathrm{~h}$ and scanned by a highresolution microcomputed tomography system ( $\mu \mathrm{CT}-100$; Scanco Medical AG, Switzerland) when the image resolution set to $14.8 \mu \mathrm{m}(70 \mathrm{kV}$ and $200 \mu \mathrm{A}$ radiation source with a $0.5 \mathrm{~mm}$ aluminum filter). Radiographic images were produced using evaluation provided by Scano Medical AG. The bone-implant contacting area percentage (BIC) in the threads along the surface of implant, the percentage of bone volume/total tissue volume on the inner of the threads (BV/TV), the thickness of trabecular bone (Tb.Th), the average spacing between each trabecular bone (Tb.Sp) and the number of trabecular bone (Tb.N) were measured.

\subsection{Specimen preparation and histological evaluation}

After micro-CT analysis, the specimens were stained with Gieson picro fuchsin and Stevene blue as previously reported. ${ }^{29}$ In short, tissues were dehydrated and then embedded in methyl methacrylate, sectioned into $10 \mu \mathrm{m}$ slices using a micro grinding system (Exart 400CP, Exakt Apparatebau) and a diamond band saw (Exakt $300 \mathrm{CP}$, Exakt Apparatebau). The BIC percentage and the BV/TV were measured.

\subsection{Specimen preparation and histomorphometric analysis}

Tissues involving implants were retrieved and fixed in $4 \%$ paraformaldehyde solution for $24 \mathrm{~h}$, and immersed in $0.5 \mathrm{M}$ EDTA solution for decalcification at room temperature for 4 weeks subsequently. The implants were rotated out of the position after being demineralized. The specimens without implants were dehydrated using graded ethanol and then process of appropriate embedding with paraffin was proceeded. After that, tissues were sectioned into $4 \mu \mathrm{m}$ slices. The slices were immunohistochemically stained for histological analysis. Antibodies to Runx2 (ab23981, Abcam), antibodies to Osterix (ab22552, Abcam), and antibodies to BMP-2 (ab14993, Abcam) were used as primary antibodies. All sides of slides were incubated with primary antibodies at $4{ }^{\circ} \mathrm{C}$ overnight. After that, the secondary antibody (Abcam) was incubated for $50 \mathrm{~min}$ at room temperature. Photomicrographs were taken using an optical 
microscope when the slices were 40 times magnification. The positively shaded bone area percentage (\%) was assessed using image analysis software to evaluate reactivity of antigen of each interest protein.

\subsection{Statistical analysis}

All of the statistical analyses were performed with SPSS (version 22.0; Chicago, IL, USA). All the in vitro experiment results analyses were showed as the mean and standard deviation (SD). At all the time points, a one-way ANOVA test with a SNK post hoc test followed was executed to confirm whether statistical difference between the blank group, the HA group and PTH groups existed. $P<0.05$ was taken for statistical difference.

\section{Results}

\subsection{Surface characterization analysis}

Irregular porous structures appeared on the titanium surfaces after sand blasting and acid etching (Fig. 1a). FSEM observation showed the rod-like structure anchored in pits of the surface. The spheroidal structure observed is apex of the rod which perpendicular to titanium surface. With the increase of assemble layers, the morphology of PTH became intensive but the rod-like structure remained as before.

XPS results showed that, with the accumulation of layer number, the $\mathrm{N}$ molecular intensity increased while the intensity of Ti molecular intensity decreased (Fig. 2a and b). This demonstrated that the content of the coatings increased and the titanium surface was covered gradually.
The release of PTH is shown in Fig. 2c-e. It demonstrated that different groups release pulse, and had similar releasing tendencies. When the release time was extended, the concentration increased. The PTH release of 5-layer group was less than the other two groups, and there is no significant difference between the total release of 7-layer group and 10-layer group.

In Fig. 2f, the water contact angle showed that the successive functionalization stepped on the titanium surfaces. On the titanium plate, the contact angle after the etching step was determined as $(82.3 \pm 5.5)^{\circ}$. This high angle shows a hydrophobic character on the surface of the blank group. After the hyaluronic acid coating, the mean angle decreased to (7.7 \pm $0.2)^{\circ}$. Coated with 5, 7 and 10 layers of PTH, the contact angles of the titanium surface were $(21.3 \pm 3.5)^{\circ},(24.2 \pm 5.3)^{\circ}$ and $(26.9$ $\pm 0.8)^{\circ}$, successively. This observation indicated that the PTH covering the surface had better hydrophilily than the blank group but was hydrophobic compared with the HA group. However, it still allowed a moderate wettability of the titanium plate.

\subsection{Cell proliferation}

The number of attached cells was determined to evaluate BMSCs proliferation (Fig. 3a). On the first and third day, the number of cell increased significantly on the PTH surface compared to HA surface $(P<0.05)$. However, at 7 days, BMSCs were found to proliferate on HA and all PTH surfaces without any statistically significant difference. Meanwhile, all the groups increased significantly at 3 days compared to 1 day and at 7 days compared to 3 days.
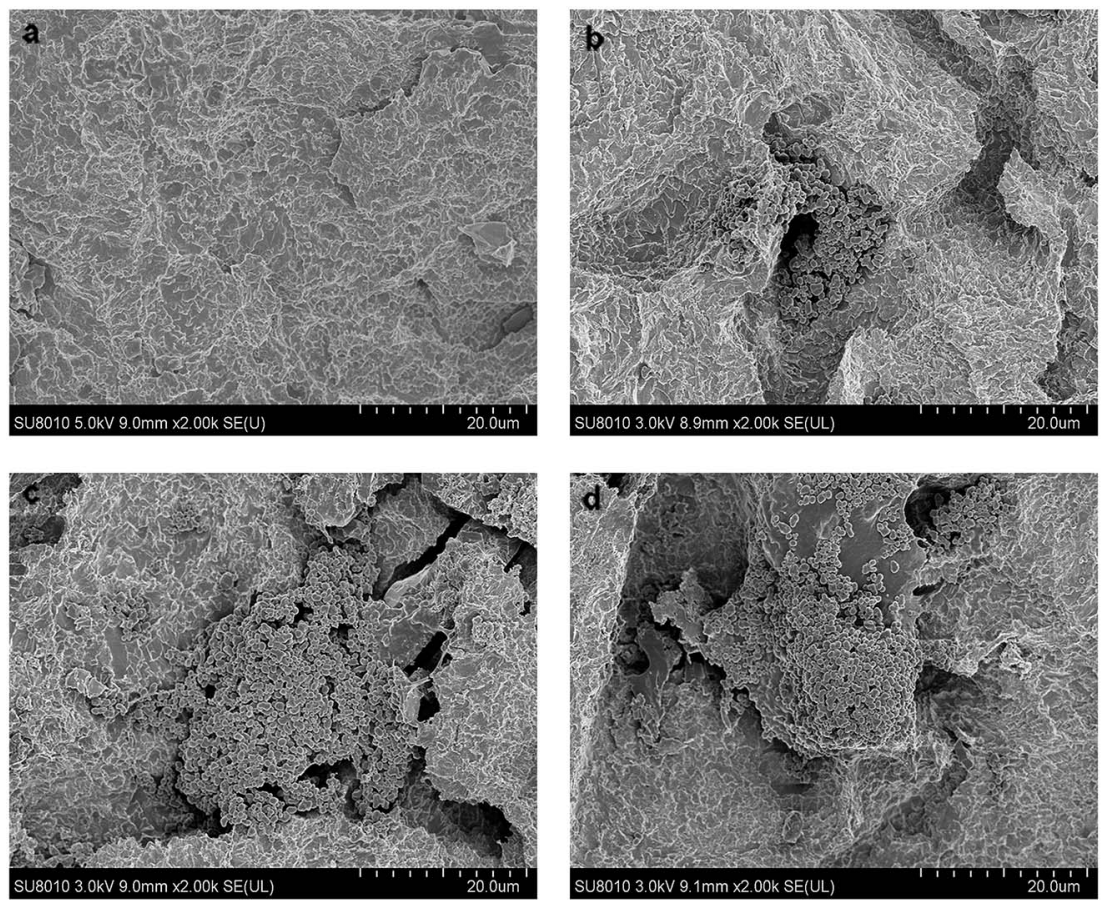

Fig. 1 Surface morphologies of the coating were detected by FSEM. (a) FSEM micrograph of the roughened titanium plates surfaces with sandblasting and etching without chitosan and HA coating. (b-d) FSEM micrographs of chitosan and HA in the rough titanium surface with 5 (b), 7 (c), and 10 (d) layers of PTH. 
a
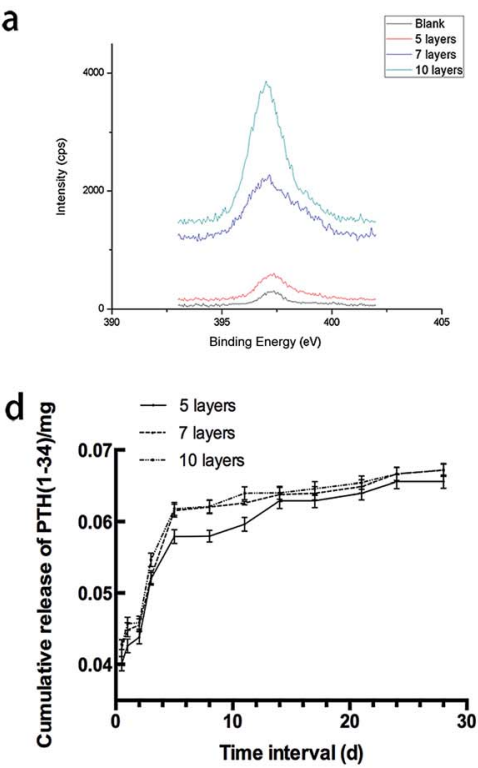

b

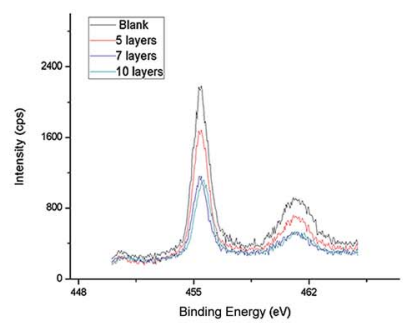

e

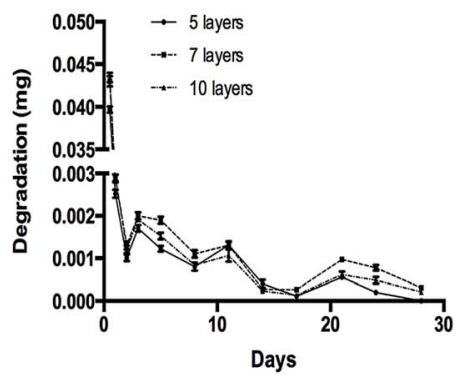

$C$

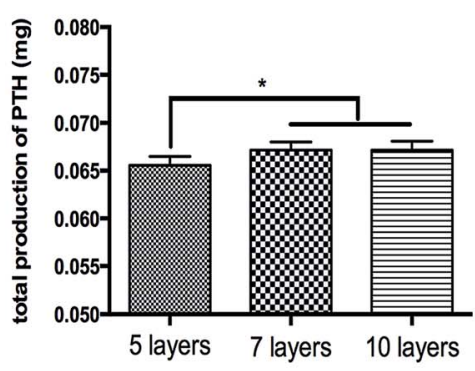

f

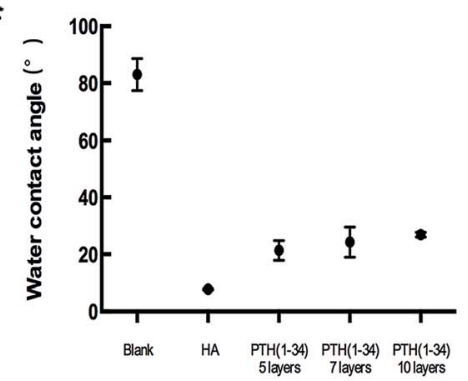

Fig. 2 Physicochemical characteristics and release concentration of the coatings were evaluated. (a) The intensity of $\mathrm{N} 1 \mathrm{~s}$ in different layers of PTH coating. (b) The intensity of Ti $2 p$ in different layers of PTH coatings. (c-e) The total release amount, the release curve (d), and the degradation curve (e) for different layers of PTH coatings. (f) The water contact angle for the blank group, control group and PTH groups.

\subsection{ALP activity}

When compared with the HA group, ALP activity in the PTH groups significantly increased. However, no significant difference was observed at day 14 between the HA group and PTH group (Fig. 3b).

\subsection{Osteocalcin secretion}

The releasing quantity of osteocalcin which was produced by BMSCs is shown in Fig. 3c. It demonstrated that 5 layers, 7 layers and 10 layers PTH significantly enhanced the osteoclacin expression $(P<0.05)$ compared with the HA group at day 7 . In addition, 7 layers and 10 layers PTH showed better ability of osteogenic differentiation than 5 layers. While at 14 days, 7 layers and 10 layers showed a significant increase compared to the 5 layers and HA group $(P<0.05)$.

\subsection{Real-time PCR analysis}

The expression of BMP-2, Runx2 and Osterix was detected to evaluate the effect on osteoblastic differentiation of BMSCs. a
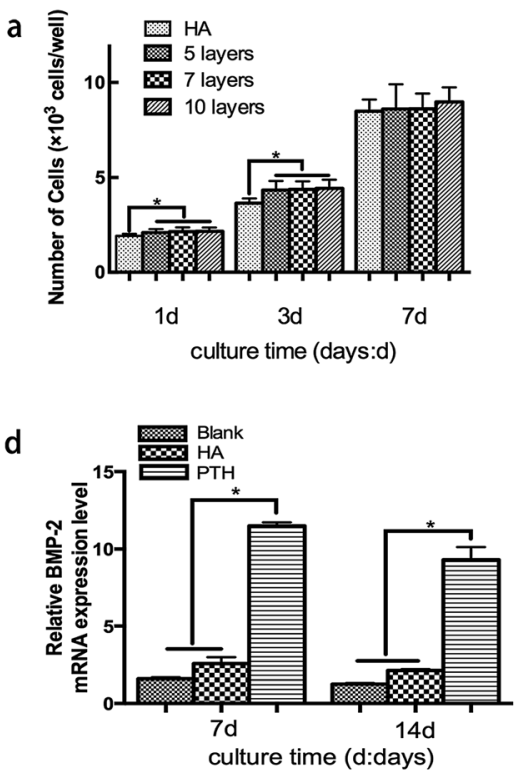

b

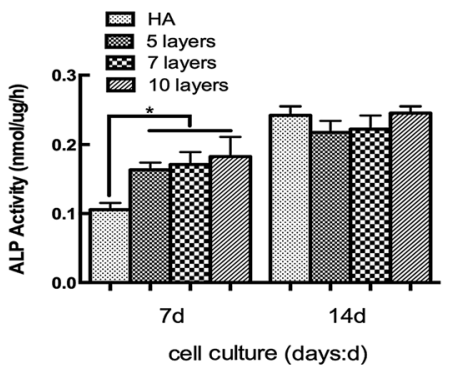

e

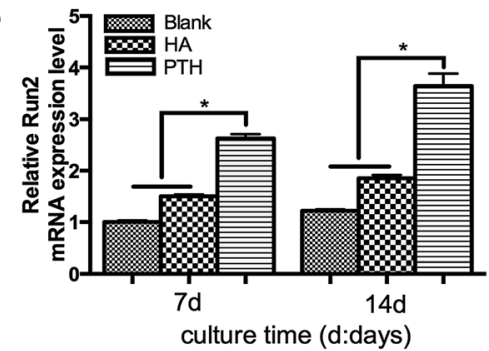

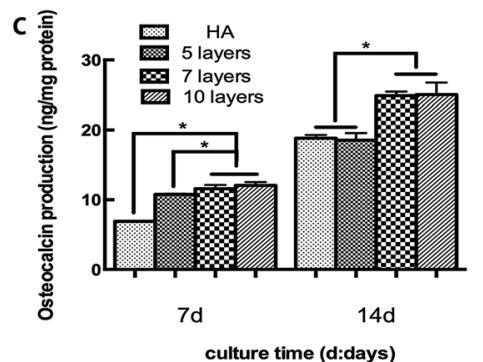

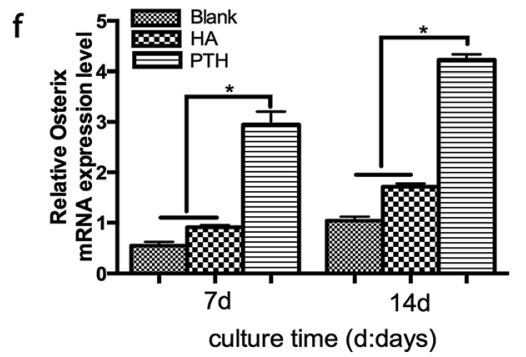

Fig. 3 Analyses of in vivo studies. (a) The number of BMSCS seeded on HA and PTH coated titanium surfaces. (b) Effects of PTH and HA coatings on the activity of intracellular ALP in BMSCS. (c) Effects of PTH and HA coatings on the production of osteocalcin in the BMSCs. (d-f) Relative mRNA expression level of BMP-2 (d), Runx2 (e), and Osterix (f) at each experiment time point. ${ }^{*}, p<0.05$ compared to the blank group or the HA group. 
According to the results of ALP activity and osteocalcin secretion analyses, 7-layer group was chosen in this analysis. BMP-2 gene was up-regulated in PTH group significantly compared with the other two groups at day 7. Although the expression of BMP-2 decreased after 14 days, PTH group still showed a higher level (Fig. 3d). Moreover Runx2 gene and Osterix gene were significantly increased in PTH group at all the time points
(Fig. 3e and f). The results indicated that PTH might have a positive effect on osteogenic response.

\subsection{Micro-CT analysis}

At 1 week and 2 weeks, implants were mainly covered with osteoid. The osteoid coverage within threads was less in the blank group and the HA group when compared with the PTH

\section{a}
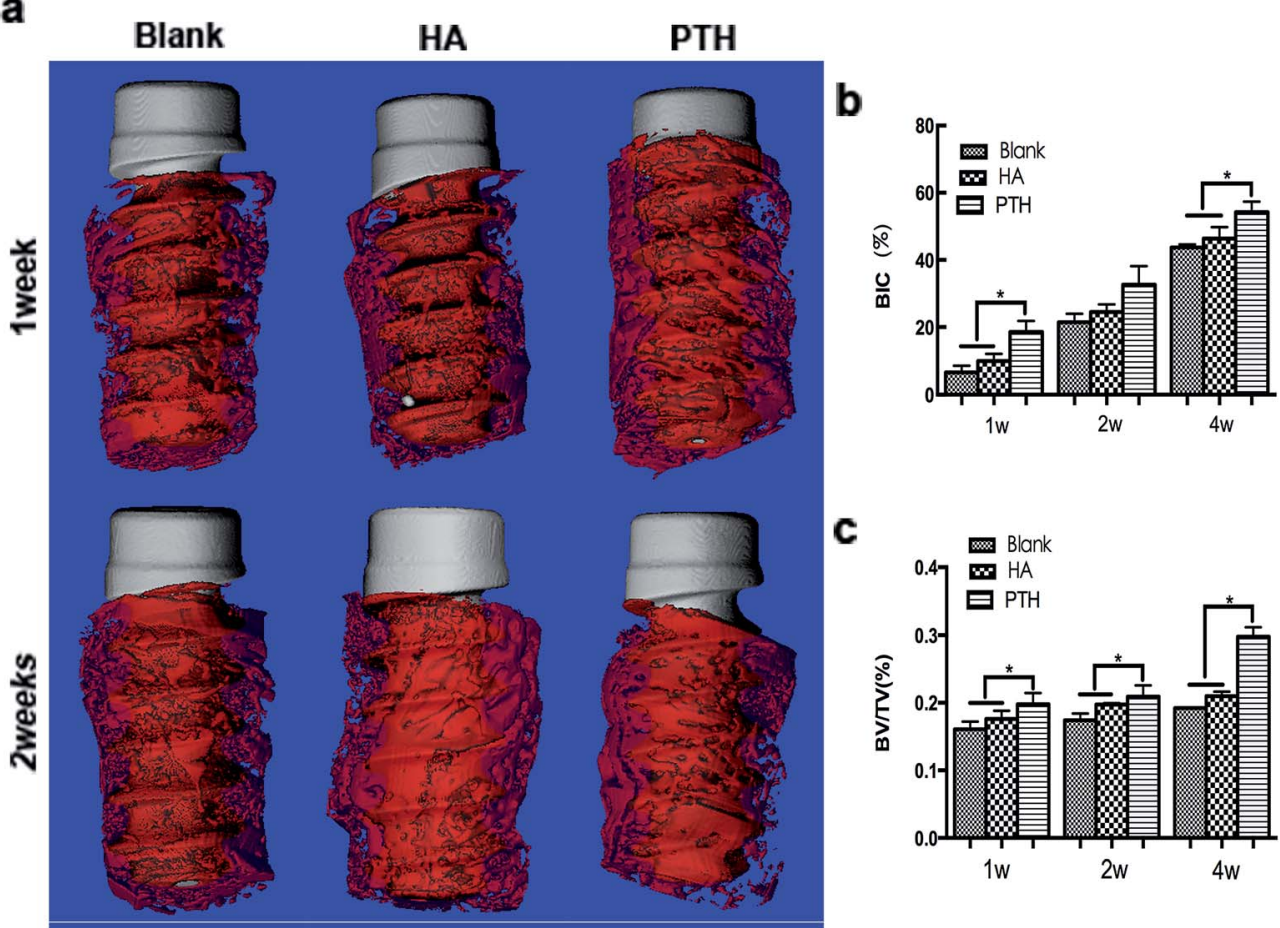

(2)

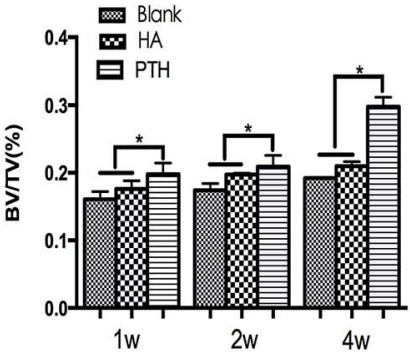

d

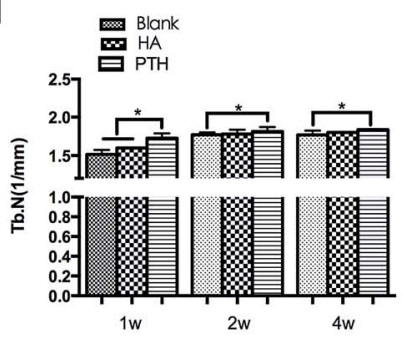

e

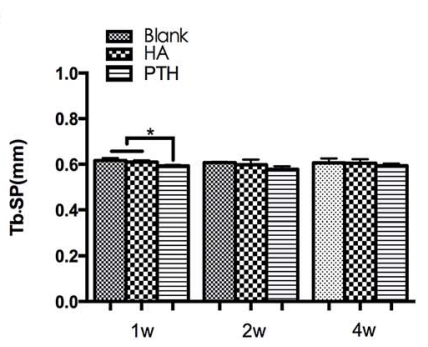

f

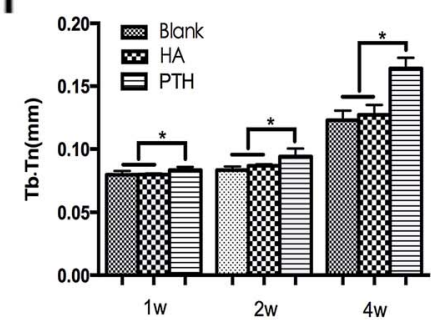

Fig. 4 New bone formation observed around the implants with micro-CT. (a) The images scanned by micro-CT showed the regeneration of bone around the implants at 1, 2, and 4 weeks. (b) Multiple-comparison analysis of BIC along the surface of implants inside the threads at all the time points treated with different groups. (c) Multiple-comparison analysis of BV/TV at all the time points treated with different groups. (d-f) Multiple-comparison analysis of Tb.N (d), Tb.Sp (e), and Tb.Th (f) at all the time points treated with different groups. $n=6$ in each of the groups. *, $p<0.05$ vs. the blank group or the HA group. Scale bar $=1 \mathrm{~mm}$ (a). 
group. At 4 weeks, new bone developed. The PTH group demonstrated to have more mature bone tissue than the blank group or the HA group (Fig. 4a).

The BIC along the surface of implants inside the threads were evaluated as well as the stability of implants in the early stage. At 1 week and 4 weeks, the BIC of the PTH group was significantly higher compared with the other two groups (Fig. 5b). The BV/TV was evaluated as well as the amount of new bone regeneration. At 1 week, 2 and 4 weeks, the BV/TV of the PTH group was significantly higher compared with the other two groups (Fig. 5c). The Tb.Th, the Tb.Sp and the Tb.N were evaluated of the quality of new bone formation. At 1 week, Tb.Sp, Tb.N, and Tb.Th of PTH group had significant different when compared with blank group of HA group. But at 2 weeks and 4 weeks, only Tb.Th and Tb.N had significant difference (Fig. 4d-f).

\subsection{Histological analysis}

After 1 week, no significant differences were found between three groups. There were osteoblast-like cells in the cavities of cortical bone, suggesting that new bone began to form. There was osteoid on implant surfaces in the marrow space. After 2 weeks, the implants of the blank group and the HA group were still surrounded by osteoid. Otherwise, new bone formed on and along the implant surfaces in the PTH group. After 4 weeks, bone tissue appeared on both types of implant surfaces. Marrow cavities were sparse in new bone, indicating maturation of the new bone. However, there was more bone tissue inside the threads of the PTH group implants than the other groups (Fig. 5a).

The percentage of mean area of bone inside all of the threads is shown in Fig. 5b. A significant larger area of bone was observed in the PTH group compared to the other two groups after 1 week and, 2 and 4 weeks. Fig. 5c shows the mean percentage of BIC values along the entire surface of implants. After 1 week and 2 weeks, there was little contact along the surface of the blank group and the HA group. In the PTH group, many more areas of contact had formed between bone and the implant surfaces. After 4 weeks, the BIC of the blank group and the HA group increased notably. However, the PTH group still showed significantly greater BIC compared to the other groups.

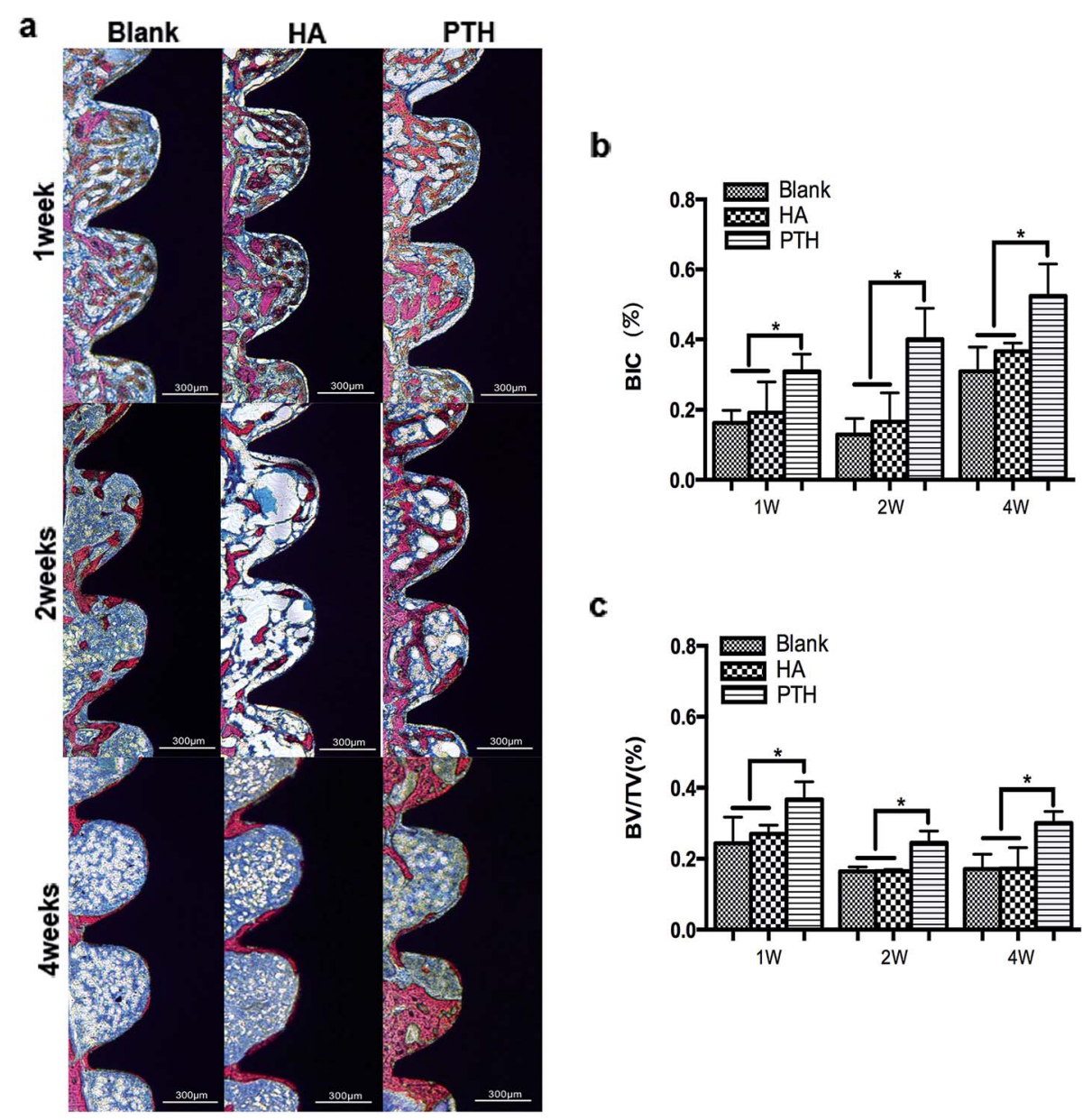

Fig. 5 Histologic sections of three groups and analysis. (a) Representative views of three groups at 1, 2, and 4 weeks. Original magnification, $\times 40$. (b) The percentage of mean area of bone inside all of the threads at each experimental point in time. (c) Mean BIC inside the threads along the surface of implants at each experimental point in time. $n=6$ in each of the group. ${ }^{*}, p<0.05$ vs. the blank group or the HA group. Scale bar $=300$ $\mu \mathrm{m}$ (a). 

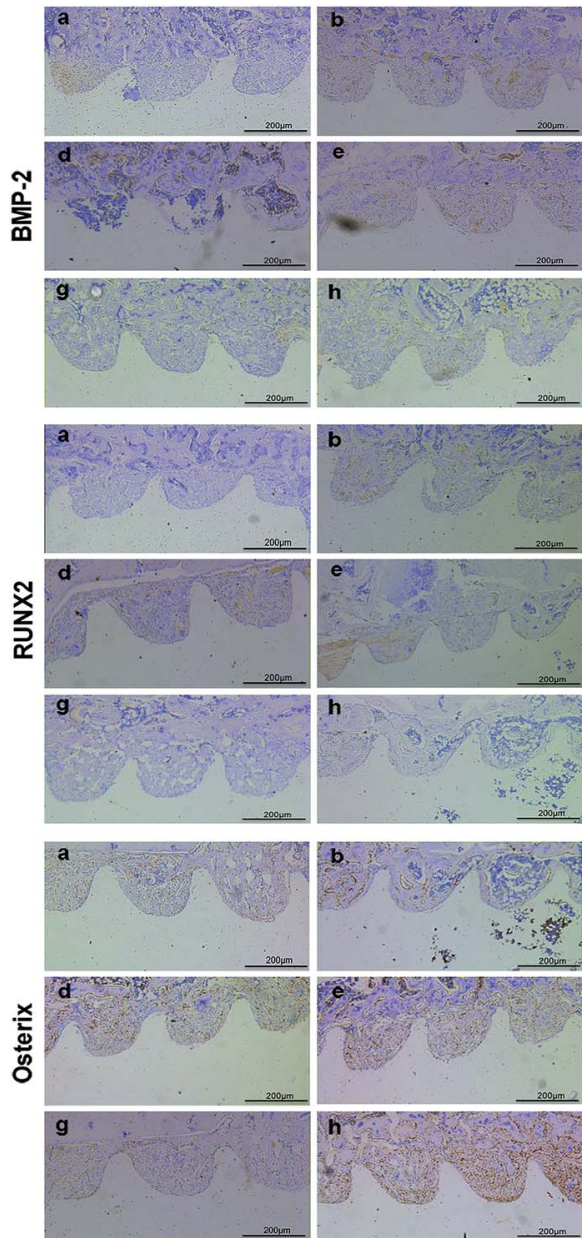
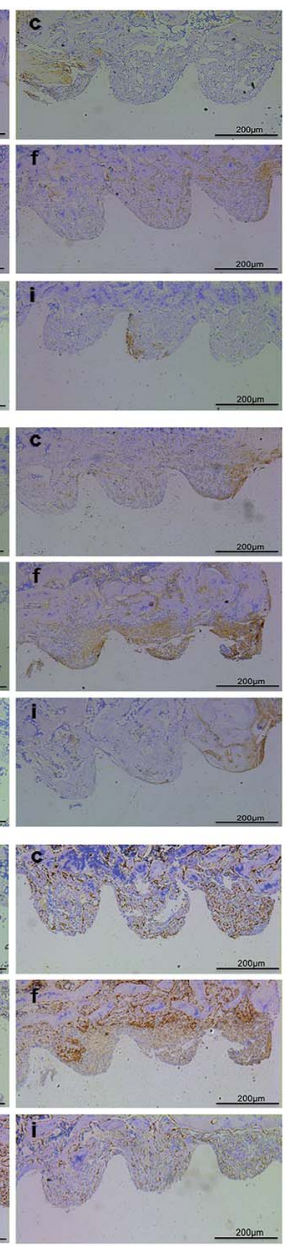

j

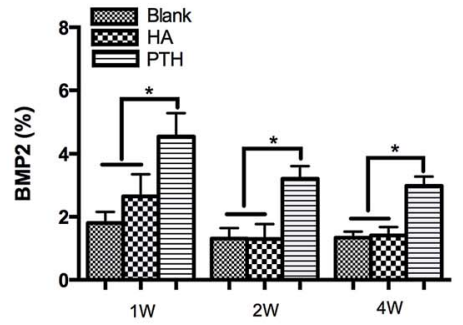

k

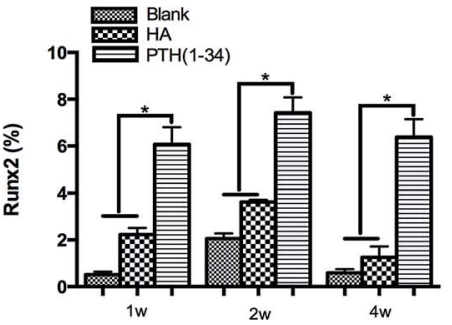

I

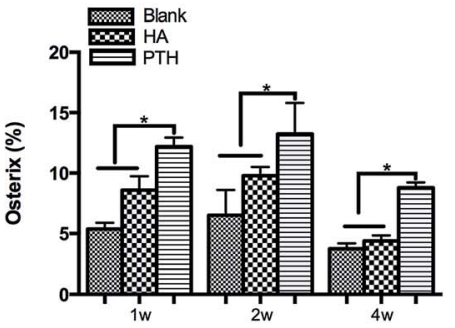

Fig. 6 Images and analyses of immunohistochemical staining for BMP-2, Runx2 and Osterix at (a-c) 1 week, (d-f) 2 weeks, and ( $g-i) 4$ weeks. (a, d, g) Blank group; (b, e, h) HA group; (c, f, i): PTH group. Original magnification, $\times 40$. (j-l) Histomorphometric staning analyses of BMP-2 (j), Runx2 (k) and, Osterix (l) antigen reactivity within the threads at each experimental point in time. $n=6$ in each of the groups. *, $p<0.05$ vs. blank group or HA group. Scale bar $=200 \mu \mathrm{m}(\mathrm{a}-\mathrm{i})$.

\subsection{Expression of Runx2, Osterix and BMP-2}

The immunohistochemical staining of Runx2, Osterix and BMP2 was showed in Fig. 6a-i. At 1 week and, 2 and 4 weeks, the positive expression of BMP-2, Runx2, and Osterix were showed significant higher levels respectively in the PTH group than in the other two groups (Fig. 6j-1).

\section{Discussion}

In this study, we demonstrated that PTH was successfully deposited on titanium surface with the LBL process, which had a positive effect on osseointegration.

Both structural and functional interrelationships between implant surfaces and bone tissues determine the success of oral implantation. The positive effect on osseointegration of different peptides was investigated in previous studies. In this context, we explored that PTH coating enhances bone-implants bonding in the early stage. The results showed PTH coating improved osseointegration resembling the other peptide.

Nakajima found that PTH can promote the BMSCs proliferation and differentiation in the early phase of bone healing. ${ }^{30}$ It reported that PTH can enhance the osteogenic differentiation of BMSCs. ${ }^{31}$ In this study, PTH enhanced proliferation in the first three days and promoted the differentiation at the later stage. This was consistent with the previous study. PTH on the titanium was almost all resealed on the 10th day. It might be the reason that there was no significant difference of ALP on the 14 th day.

In research on osteoporosis, ovariectomized rats is a mature animal model. That is the reason why this study used them to check the hypothesis. The duration of 4 weeks was chosen because this corresponded to the reparative stage when stabilized bone ends. What is more, bone around the implants matures and osseointegration begins to form at 4 weeks. The results of surface characterization and an in vivo study showed that the 7-layer structure was stable. Meanwhile, the ability to promote osteogenic differentiation was the best. Therefore, 7layer group was chosen in the in vivo study.

The results of the in vivo study suggested implants with PTH coating have a positive effect on osseointegration in the early stage. One-week implants were surrounded by osteoid which are fibres and organic matrix secreted by osteoblast and have 
a greater volume compared with mature bone. Mature bone tissue began to form after 2 weeks and the new bone tissue was sparser than osteoid. However, at each time points, the PTH group still had the best effect on promoting bone formation. This is in accordance with the results of the in vivo study that showed BMSCs treated with PTH had better osteogenic differentiation potential than the other groups. Micro-CT showed Tb.Sp of the bone decreased and the density of the trabecular improved. There might be a relation with the enhancement of mechanical strength. A previous study showed that daily administration of teriparatide led to Tb.N and Tb.Th of bone being significantly increased. ${ }^{32}$ It was reported that PTH treatment can increase implant bone contact and enhance bone density around the implant thus improving early implant fixation. ${ }^{33}$ Ryoko found that administration of PTH increased the BMD and maximum load of the lumbar vertebra and femoral shaft. ${ }^{34}$ The results of this study showed no difference with the existing study.

Because of the advantages of greater selectivity, specificity and efficacy over traditional medicines, therapeutic peptides are accepted gradually. What is more, they minimize the risks of toxicity because the metabolites of peptides are amino acids. Few peptides accumulate in bodies due to their short half-life. ${ }^{35}$ Compared with proteins and antibodies, peptides have advantages including lower costs, higher activity, and greater stability. Moreover, therapeutic peptides are generally less immunogenic. ${ }^{36}$

The rod-like structure we observed by FSEM provides a porous structure that allows relatively stable structure anchorage for bone cement to the surrounding tissue. ${ }^{36}$ The results of XPS indicated that, when achieving a high layer, protein might not be able to be assembled. The release test showed that the release concentration of 7-layer and 10-layer was almost the same. It might be due to that immersive assembly would result in inhomogeneous, interpenetrated layers. ${ }^{37} \mathrm{PTH}$ is macromolecular peptide, and arranged irregularly when assembled. With the amount of layer increase, ionic bond is not enough to assemble more PTH. Another reason might be that as the layer increase, the thickness and weight of coating increase. And when it gets the limitation, the basal layer might have collapsed. It is unable to load more PTH, even if assembly continues. However, all of these are speculations. More studies are needed in the future to solve the problem. The results of the water contact angle showed that the blank group had a hydrophobic character on the surface, while HA group was more hydrophilic than the PTH groups. However, the PTH groups had an advantage in cell adherence over the blank group. Therefore, the results on surface characteristics demonstrated that PTH incorporated with HA provided a proper structure for cell growth.

In this study, HA coating functioned as a carrier of PTH. From a clinical view, HA shows advantages on economy and convenience. The layer-by-layer assembling process was simple and PTH can successfully be incorporated into HA coatings.

PTH act on various signalling pathways including MAPK pathways, protein kinase $\mathrm{C}$ (PKC), and protein kinase A (PKA) by activating PTH1 receptor (PTH1R). ${ }^{38}$ Among them, Runx2 mediated action is the most convincing. Researchers found PTH mediated bone healing possibly via Runx2 and Osterix induction. ${ }^{39,40}$ It is believed that Runx2 is absolutely required for osteoblast development. ${ }^{\mathbf{4 1 , 4 2}}$ Osterix is known as a downstream gene of Runx2. It is required for bone formation and mineralization. In this study, the results investigated that PTH upregulated expression of Runx2 and Osterix in mRNA and protein level. Moreover, BMP-2 gene and protein expression was increased in the PTH group. However, the positive effect on BMP-2 worked in the early stage, enhancement on Runx2 and Osterix were found in the late stage. Previous study reported that BMP-2 was an upstream gene of Runx $2 .{ }^{43}$ What is more, when BMP-2 is deficient, cells fail to active Runx2/Osterix axis and osteoblastic differentiation decreases. ${ }^{\mathbf{4 4}}$ It demonstrated PTH might regulate BMP-2 signal pathway to affect bone formation, mineralization and osseointegration.

The enhancement of PTH on implant osseointegration is confirmed by far. However, regeneration of bone is a balance of osteoblast and osteoclast. Future study is needed to assess the effect of PTH on osteoclast activity.

\section{Conclusion}

It was concluded that PTH was successfully deposited into HA coatings with the layer-by-layer assembling method and PTH coatings enhanced differentiation of osteoprogenitor cells, which has the potential benefit of enhancing implant osseointegration.

\section{Conflicts of interest}

There are no conflicts to declare.

\section{Acknowledgements}

This work was supported by the health department of Zhejiang province fund (Grant No. 2014KYA127 and Grand No. 2015KYA127). We thank American Journal Expert (AJE) for its linguistic assistance during the preparation of this manuscript.

\section{Notes and references}

1 L. G. Raisz, J. Clin. Invest., 2005, 115, 3318-3325.

2 A. M. Parfitt, C. H. E. Mathews, A. R. Villanueva, M. Kleerekoper, B. Frame and D. S. Rao, J. Clin. Invest., 1983, 72, 1396-1409.

3 H. Devlin and K. Horner, J. Oral Rehabil., 2008, 35, 152-157. 4 G. Alsaadi, M. Quirynen, A. Komarek and D. van Steenberghe, J. Clin. Periodontol., 2007, 34, 610-617.

5 M. A. Bonifacio, S. Cometa, M. Dicarlo, F. Baruzzi, S. de Candia, A. Gloria, M. M. Giangregorio, M. MattioliBelmonte and E. De Giglio, Carbohydr. Polym., 2017, 166, 348-357.

6 R. Junker, A. Dimakis, M. Thoneick and J. A. Jansen, Clin. Oral Implants Res., 2009, 20(suppl. 4), 185-206.

7 N. Jiang, P. Du, W. Qu, L. Li, Z. Liu and S. Zhu, Int. J. Nanomed., 2016, 11, 4719-4733. 
8 Y. Xin, J. Jiang, K. Huo, T. Hu and P. K. Chu, ACS Nano, 2009, 3, 3228-3234.

9 Y. Huang, Q. Luo, X. Li, F. Zhang and S. Zhao, Acta Biomater., 2012, 8, 866-877.

10 C. M. Schmitt, M. Koepple, T. Moest, K. Neumann, T. Weisel and K. A. Schlegel, Clin. Oral Implants Res., 2016, 27, 13391348.

11 S. Y. Yoo, S. K. Kim, S. J. Heo, J. Y. Koak, J. H. Lee and J. M. Heo, Int. J. Oral Maxillofac. Implants, 2015, 30, 754-760.

12 V. Z. Cochenski Borba and N. C. Pietszkowski Manas, Arq. Bras. Endocrinol. Metabol., 2010, 54, 213-219.

13 E. Canalis, A. Giustina and J. P. Bilezikian, N. Engl. J. Med., 2007, 357, 905-916.

14 A. Neuprez and J.-Y. Reginster, Best Pract. Res., Clin. Endocrinol. Metab., 2008, 22, 869-883.

15 T. A. Einhorn and L. C. Gerstenfeld, Nat. Rev. Rheumatol., 2015, 11, 45-54.

16 F. A. Soliman and S. Y. Hassan, Nature, 1964, 204, 693-694.

17 Y. F. Li, X. D. Li, C. Y. Bao, Q. M. Chen, H. Zhang and J. Hu, Osteoporosis Int., 2013, 24, 1063-1071.

18 U. Kuchler, E. R. Luvizuto, S. Tangl, G. Watzek and R. Gruber, J. Dent. Res., 2011, 90, 1001-1006.

19 S. Pallu, J. C. Fricain, R. Bareille, C. Bourget, M. Dard, A. Sewing and J. Amedee, Acta Biomater., 2009, 5, 3581-3592.

20 A. Vallee, V. Humblot and C. M. Pradier, Acc. Chem. Res., 2010, 43, 1297-1306.

21 X. Yu, L. Wang, X. Jiang, D. Rowe and M. Wei, J. Mater. Sci.: Mater. Med., 2012, 23, 2177-2186.

22 A. Perez-Anes, M. Gargouri, W. Laure, H. Van Den Berghe, E. Courcot, J. Sobocinski, N. Tabary, F. Chai, J. F. Blach, A. Addad, P. Woisel, D. Douroumis, B. Martel, N. Blanchemain and J. Lyskawa, ACS Appl. Mater. Interfaces, 2015, 7, 12882-12893.

23 H. Xing, X. Wang, S. Xiao, G. Zhang, M. Li, P. Wang, Q. Shi, P. Qiao, L. E and H. Liu, Int. J. Nanomed., 2017, 12, 77097720.

24 Q. Shi, Z. Qian, D. Liu and H. Liu, Front. Physiol., 2017, 8, 574.

25 X. W. Wang, K. L. Cooper and A. Wang, in Nanocoatings, ed. G. B. Smith and M. B. Cortie, Spie-Int Soc Optical Engineering, Bellingham, 2007, vol. 6647, pp. G6470-G6470.

26 G. L. Yang, F. M. He, X. F. Yang, X. X. Wang and S. F. Zhao, J. Biomed. Mater. Res., Part A, 2009, 90, 175-185.

27 G.-l. Yang, F.-m. He, S.-s. Zhao, X.-x. Wang and S.-f. Zhao, Int. J. Oral Maxillofac. Implants, 2008, 23, 1020-1028.
28 N. Polisetti, V. G. Chaitanya, P. P. Babu and G. K. Vemuganti, Neurol. India, 2010, 58, 201-208.

29 J. Zhang, H. Wang, J. Shi, Y. Wang, K. Lai, X. Yang, X. Chen and G. Yang, Sci. Rep., 2016, 6, 23422.

30 A. Nakajima, N. Shimoji, K. Shiomi, S. Shimizu, H. Moriya, T. A. Einhorn and M. Yamazaki, J. Bone Miner. Res., 2002, 17, 2038-2047.

31 Y. Fan, J. I. Hanai, P. T. Le, R. Bi, D. Maridas, V. DeMambro, C. A. Figueroa, S. Kir, X. Zhou, M. Mannstadt, R. Baron, R. T. Bronson, M. C. Horowitz, J. Y. Wu, J. P. Bilezikian, D. W. Dempster, C. J. Rosen and B. Lanske, Cell Metab., 2017, 25, 661-672.

32 M. Sato, Y. L. Ma, J. M. Hock, M. S. Westmore, J. Vahle, A. Villanueva and C. H. Turner, J. Pharmacol. Exp. Ther., 2002, 302, 304-313.

33 R. Skripitz and P. Aspenberg, Clin. Orthop. Relat. Res., 2001, 427-432.

34 R. Takao-Kawabata, Y. Isogai, A. Takakura, Y. Shimazu, E. Sugimoto, O. Nakazono, I. Ikegaki, H. Kuriyama, S. Tanaka, H. Oda and T. Ishizuya, Calcif. Tissue Int., 2015, 97, 156-168.

35 D. J. Craik, D. P. Fairlie, S. Liras and D. Price, Chem. Biol. Drug Des., 2013, 81, 136-147.

36 D. P. McGregor, Curr. Opin. Pharmacol., 2008, 8, 616-619.

37 S. B. Kim, Y. J. Kim, T. R. Yoon, S. A. Park, I. H. Cho, E. J. Kim, I. A. Kim and J. W. Shin, Biomaterials, 2004, 25, 5715-5723.

38 S. Suttamanatwong, E. D. Jensen, J. Schilling, R. T. Franceschi, A. E. Carlson, K. C. Mansky and R. Gopalakrishnan, J. Cell. Biochem., 2009, 107, 284-292.

39 L. A. Kaback, D. Y. Soung, A. Naik, G. Geneau, E. V. Schwarz, R. N. Rosier, R. J. O'Keefe and H. Drissi, J. Cell. Biochem., 2008, 105, 219-226.

40 E. R. Sampson, M. J. Hilton, Y. Tian, D. Chen, E. M. Schwarz, R. A. Mooney, S. V. Bukata, R. J. O'Keefe, H. Awad, J. E. Puzas, R. N. Rosier and M. J. Zuscik, Sci. Transl. Med., 2011, 3, 101 ra93.

41 C. Banerjee, L. R. McCabe, J. Y. Choi, S. W. Hiebert, J. L. Stein, G. S. Stein and J. B. Lian, J. Cell. Biochem., 1997, 66, 1-8.

42 P. Ducy, R. Zhang, V. Geoffroy, A. L. Ridall and G. Karsenty, Cell, 1997, 89, 747-754.

43 Y. D. Zhang, S. C. Zhao, Z. S. Zhu, Y. F. Wang, J. X. Liu, Z. C. Zhang and F. Xue, Cell. Physiol. Biochem., 2017, 42, 1277-1293.

44 V. S. Salazar, S. Ohte, L. P. Capelo, L. Gamer and V. Rosen, Development, 2016, 143, 4352-4367. 